\title{
Variable Rate Adaptive Trellis Coded QAM for High Bandwidth Efficiency Applications in Rayleigh Fading Channels
}

\author{
Vincent K.N. Lau and Malcolm D. Macleod \\ email:knlau@lucent.com,mdm@eng.cam.ac.uk \\ Signal Processing and Communication Group, \\ Department of Engineering, University of Cambridge, CB2 1PZ, UK
}

12 September 1997

\begin{abstract}
A high bandwidth efficiency variable rate adaptive channel coding scheme, ATCQAM, is proposed. Known pilot symbols are transmitted periodically to aid demodulation. Past channel states are fed back to the transmitter with delay. Current channel state is then predicted at the transmitter to decide on the appropriate modulation mode for the current symbol. At good channel states, high level modulation is used to boost up the average throughput. At bad channel states, low level modulation is used to increase error protection. By matching the variable modulation level with a variable rate channel coder, the physical bandwidth is maintained constant. Design issues for the ATCQAM are considered. The effects of finite feedback delay, finite interleaving depth and mobile speed are investigated.
\end{abstract}

\section{Introduction}

Error correction codes have been widely used to combat the effect of Rayleigh fading in mobile radio channels. In traditional FEC schemes [1], fixed-rate codes were used which failed to explore the time varying nature of the channel. To keep the performance at a desirable level, they were designed for the average or worst case situation. In this paper, we propose and study a high bandwidth efficiency variable rate adaptive trellis coded QAM (ATCQAM) which varies the code rate and modulation level according to the channel condition. The receiver estimated the channel states and inform the transmitter through the use of a feedback link. When the channel state is good, the transmitter increases the throughput by using a higher level QAM. On the other hand, when the channel state is bad, the transmitter uses a lower level QAM to improve the error protection.

We use $M$-ary QAM since it is more energy efficient than the $M$-ary PSK constellations at large $M$. Known pilot symbols are periodically inserted at the transmitter $[2,3]$ to aid the demodulation. Performance degradations due to finite feedback delay, finite depth interleaving and mobile speed are investigated.

The paper is organized as follows. In section 2, we described design issues, different system components and the operation of the ATCQAM scheme. Simulation results are presented and discussed in section 3. Finally, in section 4, we conclude with a summary of results.

\section{Adaptive Trellis Coded QAM}

There are three main criteria leading to a good ATCQAM scheme. They are decoder complexity, rate compatibility, and constant bandwidth. They will be addressed as follows.

\subsection{Design of ATCQAM}

The simplified system block diagram of the proposed scheme is shown in fig. 1(a). Information bits are convolutionally encoded and the coded bits are mapped with the appropriate $M$-ary QAM symbol. Known pilot symbols are inserted at the transmitter periodically to aid the demodulation at the receiver. By means of an interpolation filter at the receiver, channel states in between the pilot positions are interpolated and used to demodulate the received symbols. The estimated channel states at pilot positions are fed back to the transmitter via a low noise (error protected) feedback link with certain delay. By means of an instantaneous linear prediction filter, current channel states are predicted at the transmitter and appropriate modulation modes are used for the current symbol.

By matching the adaptive channel coder and the adaptive modulator, all $M$-ary symbols have the same duration and hence the occupied bandwidth is constant. The varying instantaneous throughput is achieved by encoding a varying number of information bits per symbol.

The trellis encoder is based on a modified pragmatic TCM design [4] using a core rate $1 / 6$ encoder. Between each trellis transition, a variable number of uncoded bits are concatenated with the coded bits and mapped with the appropriate $M$-ary QAM symbol as shown in fig. 1(b). Hence, we have a trellis with a fixed number of states but a varying number of parallel branches between each transition step. The same Viterbi decoder can be used at the receiver. This reduces decoder complexity.

Suppose the estimated path (state sequence) diverged from the correct transmitted path at node 1 of the trellis dia- 
gram, the error path will have its Hamming distance ( $M$-ary) unaltered irrespective of the subsequent modulation modes. This corresponds to the rate compatibility. Therefore, the proposed adaptive pragmatic TCM satisfied the mentioned design criteria. The only disadvantage of the design is the presence of parallel branches. Although parallel branches are detrimental to the performance of the fixed rate TCM under Rayleigh fading, it is not the performance bottleneck of the ATCQAM by careful mapping of the signal constellation $[4,5]$.

\subsection{Interleaving}

Interleaving is used to convert the bursty fading into independent fading. Since a variable number of information bits are carried per trellis transition, the interleaving design is not trivial. The simplified block diagram for the interleaver is shown in fig. 1(b). Information bits are passed to the core encoder and the coded bits are interleaved ${ }^{1}$. At the time of transmission, an appropriate number of uncoded bits are drawn from the buffer. The uncoded bits and the interleaved coded bits are mapped with the appropriate $M$-ary QAM symbol. At the receiver, received symbols are de-interleaved and decoded. The uncoded bits are further re-scrambled to resume the original order.

\subsection{Channel State Interpolation}

To avoid severe degradation in QAM performance caused by fast Rayleigh fading, the transmitter inserted known pilot symbol periodically (1 pilot every $N_{p}$ symbols) to aid the receiver demodulation. The receiver stored a number of received pilot symbols and made use of an interpolation filter to estimate the fading in between the pilots.

Let $\tilde{c}_{r, i}$ be the complex fading at frame position $r$ of the $i$-th frame. Using a $2 P_{1}+1$-th order linear FIR interpolation filter, the interpolated fading at the $r$-th frame position (in between the pilot instants) of the $i$-th frame, $\tilde{z}_{r, i}$, is given by:

$$
\tilde{z}_{r, i}=\left[\tilde{c}_{r, i}+\tilde{\epsilon}_{r, i}\right]+\tilde{e}_{r, i} \quad r \in\left[1, N_{p}-1\right]
$$

Note that $\tilde{\epsilon}_{r, i}$ is modeled as a Gaussian residual noise with variance $\sigma_{\bar{\epsilon}}^{2}(r)$ due to imperfect filtering, $\tilde{e}_{r, i}$ is a Gaussian noise with variance $\sigma_{r}^{2}$. Hence, the interpolated fading estimate, $\tilde{z}_{r, i}$, can be modeled as a correct fading, $\tilde{c}_{r, i}$, corrupted with Gaussian noise, $\tilde{e}_{r, i}$ and the residual noise, $\tilde{\epsilon}_{r, i}$. The receiver use $\tilde{z}_{r, i}$ to perform match filtering and to compute the decision metrics to be used in the Viterbi decoder.

\subsection{Channel State Prediction}

It is essential for the transmitter to know the current fading instantaneously to decide on the appropriate modulation mode. The interpolation filtering, although very accurate, suffers from a processing delay of $\left(P_{1}-1\right) / 2$ pilot symbols. Hence, to obtain an instantaneous estimate of the current fading given the past fading, we use a linear predictor of order $P_{2}$.

\footnotetext{
${ }^{1}$ The two coded bits are not broken up in the interleaver.
}

\subsection{Operation of the ATCQAM}

There are 7 modulation modes in the proposed ATCQAM. They are listed as follow.

Mode 0: Throughput-1/3, 6 coded bits carried by 3 QAM symbols.

Mode 1: Throughput-1/2, 4 coded bits carried by 2 QAM symbols.

Mode 2: Throughput-1, 2 coded bits carried by 1 QAM symbol.

Mode 3: Throughput-2, 1 uncoded bits +2 coded bits carried by 18 PSK symbol.

Mode 4: Throughput-3, 2 uncoded bits +2 coded bits carried by 1 16QAM symbol.

Mode 5: Throughput- 4,3 uncoded bits +2 coded bits carried by $132 \mathrm{QAM}$ symbol.

Mode 6: Throughput- 5,4 uncoded bits +2 coded bits carried by $164 \mathrm{QAM}$ symbol.

The predicted channel state is partitioned into 7 segments with each segment corresponds to one of the above modes. Let $\frac{E_{s}}{\eta_{0}}$ be the average symbol energy to noise ratio. Mode $m$ is chosen if the predicted SNR $\in\left[\zeta_{m}, \zeta_{m+1}\right]$. Note that $\zeta_{0}=0$ and $\zeta_{7}=\infty$.

There are two different ways to operate the ATCQAM, namely the constant BER operation and the constant throughput operation. For the constant BER operation, we set the switching thresholds so as to maintain a relatively constant BER over a range of $\frac{E_{s}}{\eta_{0}}$. For simplicity, the ATCQAM is specified by a single parameter, $\zeta_{1}$.

For the constant throughput operation, we set the thresholds so as to maintain a constant average throughput. This can be achieved if $\zeta_{1} \propto \frac{E_{s}}{\eta_{0}}$.

\section{Results and Discussion}

Due to the varying throughput in the ATCQAM scheme, we use the average symbol energy to noise ratio, $\frac{E_{s}}{\eta_{0}}$, instead of the usual bit energy to noise ratio as a reference for comparison. We assumed $N_{p}=91$ (pilot period), $P_{1}=21$ (interpolation filter order), $P_{2}=16$ (prediction filter order) and $N_{u}=8$ (user per TDMA frame). The overhead due to pilot symbols is about $1 \%$. Convolutional code of constraint length 5 is used to construct the ATCQAM. Its performance is compared with that of the optimal fixed rate $\mathrm{TCM}^{2}$ Hence, the comparison is fair.

\footnotetext{
${ }^{2}$ Optimal in the sense of the maximal diversity order that could be achieved. For example, the fixed rate 8PSK-TCM has a diversity order of 3 which is the best out of constraint length 5 convolutional code.
} 


\subsection{Performance of ATCQAM in TDMA Frame}

Zero Feedback Delay $(\Delta=0)$ : As an illustration, we assumed zero feedback delay, $100 \times 500$ block interleaving and $f d_{T} s=1 \times 10^{-33}$. The BER and the throughput of the ATCQAM-TDMA scheme using constant BER controls are shown in fig. 2(a) and (b). Under the constant $B E R$ control, the BER remains approximately constant when $\frac{E_{s}}{\eta_{0}}$ is within the range of adaptation as shown in fig. 2(a). The level of BER and the adaptation range depends on the initial control threshold, $\zeta_{1}$. Along the BER curves of the ATCQAM, the throughput varied as $\frac{E_{s}}{\eta_{0}}$ as illustrated in fig. 2(b). At high $\frac{E_{s}}{\eta_{0}}$, we trade BER with a higher throughput. To compare with the performance of the fixed rate TCM, we have to consider the relative throughput gain of the ATCQAM at the same $\frac{E_{s}}{\eta_{0}}$ and BER. The BER against the relative throughput gains of the ATCQAM w.r.t. the fixed rate 8PSK-TCM and 16QAM-TCM are plotted in fig. 3(a). For example, at $\bar{P}_{b}=10^{-4}$, the throughput gains relative to the 8PSK-TCM and the 16QAM are 1.95 and 1.54 times respectively. However, when compared to QPSK-TCM, there is negligible gain as opposed to the results in [7] under ideal conditions. This is because of the degraded gains in the ATCQAM due to errors in the channel state estimation and prediction.

On the other hand, when operating under the constant throughput control, the throughput of the ATCQAM is maintained at 2 and 3 for comparison with fixed rate 8PSK-TCM and 16QAM-TCM. The $\frac{E_{s}}{\eta_{0}}$ gain of the ATCQAM relative to 8PSK-TCM and 16QAM-TCM is plotted in fig. $3(\mathrm{~b})$. For example, there are $7.1 \mathrm{~dB}$ and $9.3 \mathrm{~dB}$ gains in $\frac{E_{s}}{T_{0}}$ at $\bar{P}_{b}=10^{-4}$ relative to 8PSK-TCM and 16QAM-TCM respectively. Similarly, there is negligible gain relative to QPSK-TCM due to the same reason.

Effects of Finite Feedback Delay: The $\bar{P}_{b}$ of the ATCQAM under finite feedback delay at $f_{d} T_{s}=1 \times 10^{-3}$ and $f_{d} T_{s}=6 \times 10^{-3}$ are shown in in fig. $4(\mathrm{a})$ and (b) respectively with $\frac{E_{s}}{\eta_{0}}=60$ and $\bar{\zeta}=3$. The ATCQAM schemes (FDMA and TDMA), are robust to the feedback delay at slow fading (see fig. 4(a)). For example, at $\Delta=40$, $\bar{P}_{b}$ of the ATCQAM schemes are approximately 30 times smaller than the BER of the fixed rate 16QAM.

At higher fading rate (see fig. 4(b)), the ATCQAMTDMA become more sensitive to the feedback delay. At $\Delta=40, \bar{P}_{b}$ for the ATCQAM-TDMA is the same as the fixed rate $B E R$.

Effects of Block Interleaving: Fig. 5(a) and (b) shows $\bar{P}_{b}$ and $\bar{\eta}$ against $\frac{E_{s}}{\eta_{0}}$ for the ATCQAM-TDMA scheme and the fixed rate $8 \mathrm{PSK}-\mathrm{TCM}$ at various interleaving depths. $\bar{P}_{b}$ increases gradually as the interleaving depth decreases. However, at all interleaving depths, although the absolute $\bar{P}_{b}$ degrades, the ATCQAM-TDMA scheme

${ }^{3}$ This corresponds to a mobile speed of $24 \mathrm{~km} / \mathrm{hr}$ and baud rate of 40kbaud. always out-performs the corresponding fixed rate codes. For example, at $\bar{P}_{b}=2^{-3}$ with $100 \times 100$ interleaving, the gain in $\frac{E_{s}}{\eta_{0}}$ is $8.7 \mathrm{~dB}$ w.r.t. 8 PSK-TCM. At $\bar{P}_{b}=4 \times 10^{-3}$ with $35 \times 35$ interleaving, the gain in $\frac{E_{s}}{\eta_{0}}$ is $10 \mathrm{~dB}$. Hence, the proposed ATCQAM also shows significant gains in systems with small interleaving depth.

Effects of Mobile Speed: Fig. $6\left(\right.$ a) shows $\bar{P}_{b}$ against $\frac{E_{s}}{\eta_{0}}$ for the ATCQAM-TDMA scheme and the fixed rate 16QAM-TCM at $f_{d} T_{s}=6 \times 10^{-3}$ It is apparent that an irreducible error floor at $\bar{P}_{b}=3 \times 10^{-4}$ appears. Note that above the error floor, ATCQAM always outperforms the fixed rate codes.

The reason of the presence of the error floor is because of aliasing. At this high fading rate, the Nyquist sampling criteria is exceeded and aliasing causes severe degradation in the interpolation process at the receiver. This is particularly serious for high density signal constellations like 64QAM. Hence, $\sigma_{0}$, is non-zero even in the absence of channel noise and this causes the irreducible error floor. This can be avoided by reducing the pilot period at the expense of increased pilot symbol overheads.

\subsection{Performance of ATCQAM in FDMA Frame}

The ATCQAM-FDMA behaves in a similar way as the ATCQAM-TDMA. However, they do have some differences in terms of performance.

Tx-Rcv Synchronization: As discussed in section 2, only quasi-closed loop control can be applied to maintain the synchronization of the transmitter and the receiver by synchronizing the feedback delay at the receiver. This increase the complexity. On the other hand, closed loop control by embedding a control word in the frame can be used in ATCQAM-TDMA. This simplified the synchronization task at the expense of increased overheads.

Feedback Delays and BER Performance: Under most circumstances, BER for the ATCQAM-TDMA is always lower than the FDMA counterpart. Their difference exagerates at high fading rate (see fig. 4(a) and (b)). At $f_{d} T_{s}=6 \times 10^{-3}$, BER of the ATCQAM-FDMA is even higher than that of the fixed rate 16QAM at all $\Delta$. Hence, the ATCQAM-FDMA is totally not effective at $f_{d} T_{s}=6 \times 10^{-3}$.

This is due to the difference in their prediction errors. The prediction filter for the TDMA scheme only has to predict the channel states for part of the physical frame (assigned timeslot) at the vicinity of the pilot symbol. This is more accurate compared with the FDMA system where the channel states across the whole physical frame have to be predicted.

The relative prediction errors at the transmitter for the ATCQAM-TDMA scheme and the ATCQAM-FDMA schemes are shown in fig. 7 (a) and (b). As seen from the figures, the predicted channel states for the FDMA 
system is less accurate. It is more likely for symbols to be transmitted using an unsuitable mode for the FDMA system. Hence, its BER degrades. As the fading rate increases, the difference in their prediction errors increases and this enlarges their differences in $\bar{P}_{b}$.

Irreducible Error Floor: At high fading rate, irreducible error floor appears in the BER curves. The error floor depends on the fading rate. However, at the same fading rate, we found that ATCQAM-TDMA always has a lower error floor than ATCQAM-FDMA. This is illustrated in fig. 6(b). At $f_{d} T_{s}=6 \times 10^{-3}$, the error floors of the ATCQAM-TDMA and the ATCQAM-FDMA are $3 \times 10^{-4}$ and $2 \times 10^{-3}$ respectively. This means that ATCQAM-TDMA is more robust to high fading rate as well.

\section{Conclusions}

Seven modes ATCQAM scheme is proposed to exploit the time varying nature of the mobile radio channel. By means of an instantaneous linear prediction filter, current channel states are predicted at the transmitter and appropriate modulation modes are used for the current symbol. Two ways of operation of the ATCQAM, namely the constant BER operation and the constant throughput operation, are introduced.

Two frame formats, namely the TDMA and the FDMA formats, for the ATCQAM scheme are considered. Two different methods to maintain transmitter-receiver synchronization, namely the quasi-closed loop control and the closed loop control, are discussed. Under normal operating conditions, the ATCQAM has relative throughput gains around 1.5-1.9 times and relative $\frac{E_{s}}{\eta_{0}}$ gains around $7-9 \mathrm{~dB}$ w.r.t. the fixed rate codes at $\bar{P}_{b}=10^{-4}$. The effects of finite feedback link delay, finite depth interleaver and mobile speed are considered. It is found that ATCQAM-TDMA is in general more robust than ATCQAM-FDMA. At $f_{d} T_{s}=6 \times 10^{-3}$, irreducible error floor occurs but the performance of the ATCQAM-TDMA is degraded less than the ATCMQAM-FDMA.

\section{References}

[1] J. G. Proakis, Digital Communications. McGraw Hill International Editions, NY, third ed., 1995.

[2] J. K. Cavers, "An Analysis of Pilot Symbol Assisted Modulation for Rayleigh Fading Channels," IEEE Trans. on Vehicular Tech., vol. 40, pp. 686-693, Nov. 1991.

[3] S. Sampei and T. Sunaga, "Rayleigh Fading Compensation for QAM in Land Mobile Radio Communications," IEEE Trans. on Vehicular Tech., vol. 42, pp. 137-147, May. 1993.

[4] A. J. Viterbi, J. K. Wolf, E. Zehavi, and R. Padovani, "A pragmatic approach to trellis-coded modulation," IEEE Communs. Magazine, pp. 11-19, July 1989.

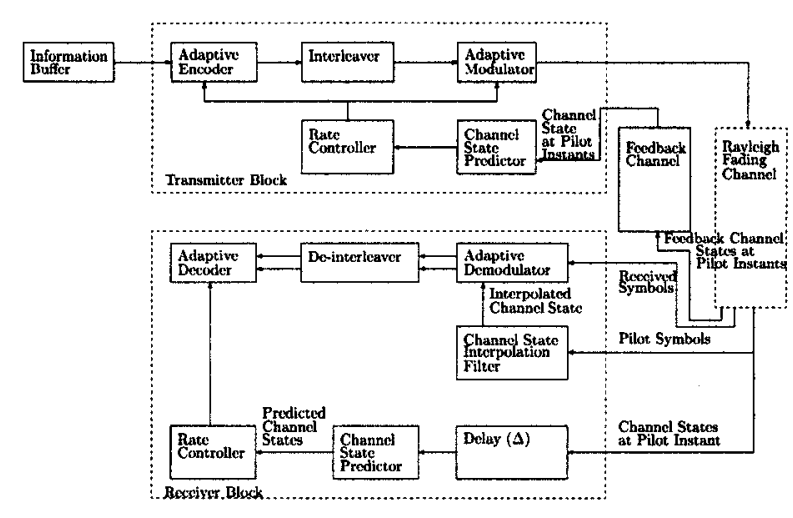

(a) Simplified Block Diagram of the ATCQAM Scheme.

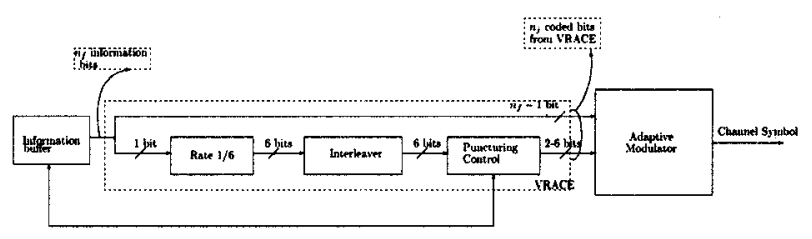

(b) Block Diagram for Variable Rate Interleaver.

Figure 1: Overall Block Diagrams of the ATCQAM Scheme.

[5] K. N. Lau and M. D. Malcolm, "Variable Rate Adaptive Trellis Coded QAM for High Bandwidth Efficiency Applications under Rayleigh Fading Channel," Submitted to IEEE Trans. on Communs.

[6] C. W. Therrien, Discrete Random Signals and Statistical Signal Processing. Prentice Hall, NJ, first ed., 1992.

[7] S. Alamouti and S. Kallel, "Adaptive Trellis-Coded Multiple-Phase-Shift Keying for Rayleigh Fading Channels," IEEE Trans. on Communs., vol. 42, pp. 2305-2314, June 1994. 


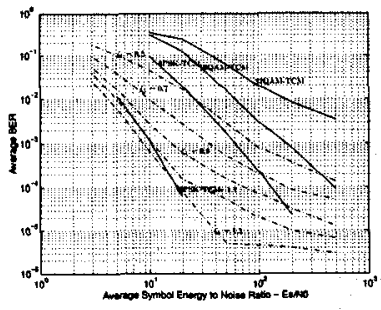

(a) Average BER against SNR at various $\zeta_{1}$ under the constant BER operation.

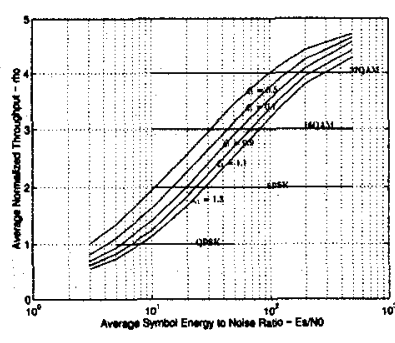

(b) Average Normalized Throughputs against SNR at various $\zeta_{1}$.

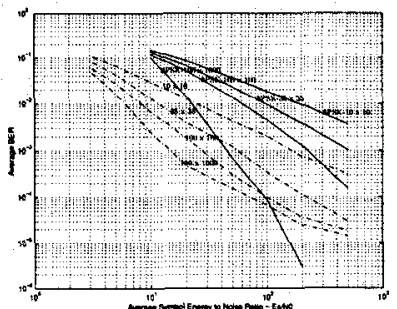

(a) $\bar{P}_{b}$ against $\frac{E_{s}}{\eta_{0}}$ at various interleaving depths.

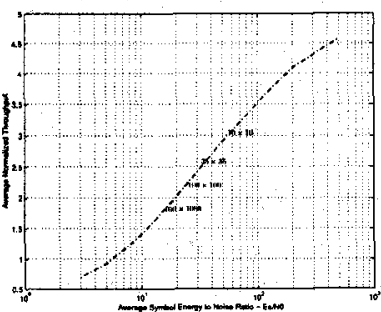

(b) $\bar{\eta}$ against $\frac{E_{s}}{\eta_{0}}$ at various interleaving depths for ATCQAM-TDMA.
Figure 2: Performance of ATCQAM-TDMA relative to 8PSK-TCM and 16QAM-TCM. Dotted lines represent ATCQAM. Solid lines represent fixed rate codes.

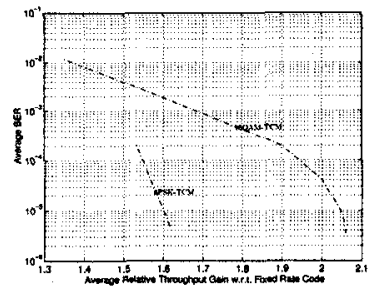

(a) Relative throughput gains of ACTQAM w.r.t. 8PSK-TCM and 16QAM-TCM.

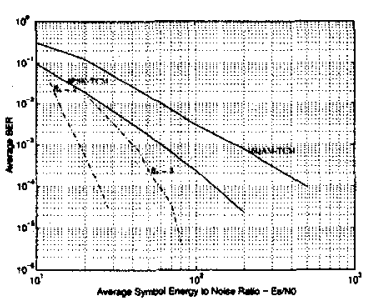

(b) Relative $\frac{E_{s}}{\eta_{0}}$ gains of the ATCQAM w.r.t. 8PSK-TCM and 16QAM-TCM.
Figure 3: Normalized performance of ATCQAM-TDMA relative to 8PSK-TCM and 16QAM-TCM. Dotted lines represent ATCQAM. Solid lines represent fixed rate codes.

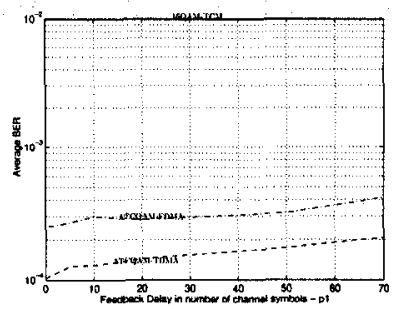

(a) Effects of feedback delay on the BER of the ATCQAM-TDMA at $f_{d} T_{s}=10^{-3}$. ATCQAM-FDMA and

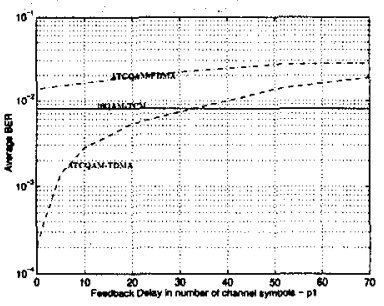

(b) Effects of feedback delay on the BER of the ATCQAM-FDMA and ATCQAM-TDMA at $f_{d} T_{s}=6 \times 10^{-3}$.
Figure 4: Effects of Feedback Delay on the performance of the ATCQAM.
Figure 5: Effects of the Interleaving Depths on the performance of the ATCQAM.

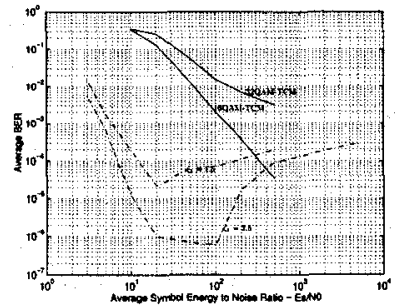

(a) BER of the ATCQAM-TDMA at $f_{d} T_{s}=6 \times 10^{-3}$.

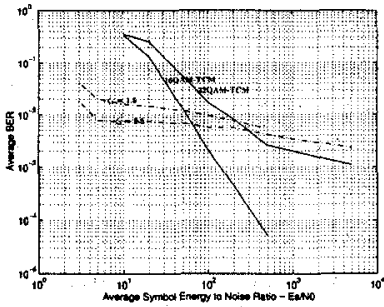

(b) BER of the ATCQAM-FDMA at $f_{d} T_{s}=6 \times 10^{-3}$
Figure 6: Effects of mobile speed and the fading rate on the BER performance of the ATCQAM-FDMA and ATCQAMTDMA schemes.

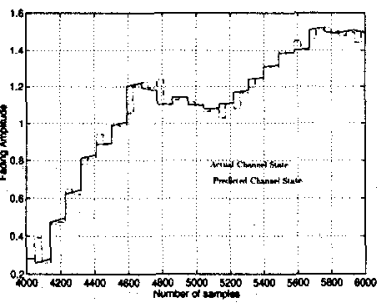

(a) Predicted channel states (dotted line) and actual channel states (FDMA).

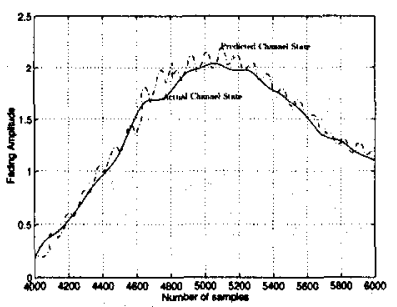

(b) Predicted channel states (dotted line) and actual channel states (TDMA).
Figure 7: Illustration of the difference in prediction error between the ATCQAM-TDMA and the ATCQAM-FDMA system. 